\title{
Guarda responsável de animais de estimação na área urbana do município de Pelotas, RS, Brasil
}

\author{
Responsible pet animal guardianship in the urban area of the \\ municipality of Pelotas in the state of Rio Grande do Sul, Brazil
}

Lídice Rodrigues Domingues ${ }^{1}$

Juraci Almeida Cesar ${ }^{1}$

Anaclaudia Gastal Fassa ${ }^{1}$

Marlos Rodrigues Domingues ${ }^{2}$

${ }^{1}$ Programa de PósGraduação em Epidemiologia, Universidade Federal de Pelotas (UFPel). Av. Duque de Caxias $250 / 3^{\circ}$, Fragata. 96030-002 Pelotas RS

Brasil.

dungavet@vetorial.net

${ }^{2}$ Programa de PósGraduação em Educação Física, UFPel.

\begin{abstract}
The study sought to estimate the presence of cats and dogs in households in the urban area of the city and assess responsible guardianship and associated factors. A population-based, cross-sectional study was conducted using the multi-stage sampling process and the census sectors of the demographic census of the year 2010 carried out by IBGE were used as conglomerates. The unit under study was the household. The outcome was responsible guardianship, defined by a score that took into account the following variables: sterilization, anti-rabies vaccination, veterinary medical consultation, control of endoparasites, access of animals to public areas, control of ectoparasites and the collection of feces of animals in public areas. Of the 1,558 households investigated, 58.9\% had pets (cat or dog). The study reveals that the score of responsible guardianship increases with the education level of the head of the household and is highest among apartment dwellers. The majority of the households investigated met only half of the criteria. The results of this study point to the need for public policies that instruct the population on responsible guardianship, and that offer immunizations and sterilization services for animals.
\end{abstract}

Key words Ownership, Pet, Animal care, Crosssectional studies, Veterinary medicine
Resumo O presente estudo teve por objetivo estimar a presença de cães e gatos em domicílios da zona urbana do município e avaliar a guarda responsável e seus fatores associados. Foi realizado um estudo transversal, de base populacional com processo de amostragem em múltiplos estágios e com os setores censitários do censo demográfico do ano de 2010, realizado pelo IBGE, utilizados como conglomerados. A unidade em estudo foi o domicílio. O desfecho foi a guarda responsável, definida por um escore que levou em consideração as seguintes variáveis: esterilização, vacinação antirrábica, consulta médica veterinária, controle de endoparasitas, acesso dos animais às áreas públicas e o recolhimento das fezes nestes locais e controle de ectoparasitas. Entre os 1.558 domicílios investigados, $58,9 \%$ possuíam animais de estimação (cão elou gato). O estudo revela que o escore de posse responsável aumenta com a escolaridade do chefe da família e é maior entre os moradores de apartamento. A maioria dos domicílios investigados atendeu apenas a metade dos critérios. Os resultados deste estudo apontam para a necessidade de políticas públicas que orientem a população sobre a guarda responsável e que ofertem serviços de imunizações e esterilização para os animais.

Palavras-chave Posse, Animais de estimação, Cuidado animal, Estudos transversais, Medicina veterinária 


\section{Introdução}

Atualmente $60 \%$ dos domicílios brasileiros possuem, pelo menos, um animal de estimação, o que representa cerca de 32 milhões de cães e 16 milhões de gatos domiciliados. Estas cifras conferem ao Brasil o segundo lugar mundial em número absoluto de cães e gatos, atrás apenas dos Estados Unidos ${ }^{1}$. Segundo a ABINPET (Associação Brasileira da Indústria de Produtos para Animais de Estimação), a estimativa é de que o chamado “mercado pet" movimente aproximadamente R \$ 14 bilhões em 2012 . Estima-se que, em 2013, o Brasil alcance o segundo lugar mundial em termos de gastos com alimentação de cães e gatos ${ }^{2}$.

O convívio e o vínculo afetivo com animais de estimação podem trazer inúmeros benefícios aos humanos. Esta interação tende a reduzir os níveis de ansiedade e assim, diminuir o aparecimento, o agravamento ou a progressão de doenças relacionadas ao estresse ${ }^{3}$. Segundo o Instituto Nacional dos Estados Unidos, estudos sobre saúde das populações deveriam considerar como uma variável importante a presença ou a ausência de animais nos domicílios bem como a natureza desta interação ${ }^{4}$. Entretanto, a crescente aquisição de cães e gatos como animais de companhia, aliada ao fato dos animais de estimação frequentarem áreas públicas, tem aumentado o número de pessoas expostas ao risco de contrair infecções por parasitos zoonóticos ${ }^{5,6}$. Pelo menos, $60 \%$ das doenças infecciosas que afetam os seres humanos e cerca de $75 \%$ de doenças novas ou emergentes, em todo o mundo, são consideradas zoonoses ${ }^{7}$.

Segundo a Organização Mundial da Saúde (OMS), o convívio com animais requer cuidados que, se ignorados, podem trazer consequências indesejadas como doenças, além de agressões, acidentes de trânsito e poluição ambiental ${ }^{8}$.

A guarda de animais de estimação vem sendo descrita em diferentes partes do mundo em relação a diversos determinantes sociais e comportamentais como o nível educacional, a classe social e a composição familiar ${ }^{9,10}$. Entretanto, pouco se sabe sobre a população de animais domésticos e a guarda responsável na realidade brasileira. Assim, este estudo objetivou estimar a presença de cães e gatos em domicílios localizados na área urbana do município de Pelotas, RS, Brasil, e avaliar a guarda responsável destes animais e seus fatores associados.

\section{Metodologia}

Foi realizado um estudo transversal de base populacional, no período de fevereiro a junho de 2012, em Pelotas (RS). O trabalho foi vinculado ao Programa de Pós-Graduação em Epidemiologia da Universidade Federal de Pelotas (UFPel) na forma de Consórcio de Pesquisa ${ }^{11}$ e investigou uma série de desfechos em saúde.

Uma equipe, de 30 entrevistadoras, foi treinada por 40 horas, para aplicação de um questionário nos domicílios amostrados. Logo após o treinamento foi realizado um estudo piloto em dois setores censitários não incluídos na amostra.

A unidade em estudo foi o domicílio e para o cálculo amostral foi utilizado o software EpiInfo 6.0. A partir da estimativa de $60 \%$ de domicílios com animais de estimação, aceitando-se uma margem de erro de 4 pontos percentuais e nível de confiança de 95\%, o tamanho da amostra, calculado para o estudo, foi de 575 domicílios. Para o estudo de fatores associados, assumindo-se esta mesma prevalência (60\%), erros alfa e beta de 0,05 e 0,20, respectivamente, efeito de delineamento de 2,0 , somados a $10 \%$ para eventuais perdas e $15 \%$ para controle de fatores de confusão, o tamanho mínimo de amostra deveria ser de 716 domicílios. Por ser um estudo vinculado a uma coleta de dados em saúde pública de maior abrangência, o número final de domicílios sorteados para a investigação foi de 1.723 , superando o exigido pelo cálculo amostral. Não foi realizado cálculo de poder para o desfecho por se tratar de uma variável ordinal. O processo de amostragem foi realizado em múltiplos estágios e os setores censitários delimitados pelo censo demográfico do ano de 2010, realizado pelo IBGE ${ }^{12}$, foram utilizados como conglomerados.

A pesquisa foi realizada através da aplicação de um único instrumento contendo questões sobre os diferentes temas em estudo. As questões relativas à pesquisa sobre animais de estimação foram respondidas por apenas um morador, preferencialmente a(o) dona(o) da casa, juntamente com as demais questões gerais sobre o domicílio. As variáveis independentes estudadas foram: escolaridade do chefe da família e renda familiar no primeiro nível, tipo de moradia e número de moradores do domicílio no segundo nível e a presença de criança de até 5 anos e/ou presença de idoso no domicílio no terceiro e último nível. 
O desfecho em estudo foi a guarda responsável de cães e/ou gatos. Por não haver um instrumento padronizado para medir o desfecho em estudo, nesta pesquisa a guarda responsável foi definida através de um escore composto pelo somatório de pontos das seguintes variáveis: a esterilização, a vacinação antirrábica nos últimos 12 meses, a consulta médica veterinária nos últimos 12 meses, o controle de endoparasitas nos últimos seis meses, o acesso dos animais às áreas públicas, o controle de ectoparasitas nos últimos seis meses e o recolhimento de fezes dos animais em áreas públicas. Para cada uma das variáveis foram atribuídos os seguintes valores: às primeiras cinco variáveis foi atribuído zero para a resposta nenhum animal, um ponto para a resposta alguns animais e dois pontos para a resposta todos os animais. Para a variável controle de ectoparasitas foi atribuído zero na ausência de controle, um ponto para o controle somente no animal ou no ambiente e dois pontos para o controle em ambos. Para a variável recolhimento de fezes foi atribuído zero quando o recolhimento não era realizado, um ponto para a resposta às vezes e dois pontos para a resposta sempre ou quando o animal não sai de casa, conforme mostra o Quadro 1. Durante as análises, o escore criado foi tratado como uma variável discreta.

A análise dos dados foi feita com o software Stata 11.2 e primeiramente foi realizada uma descrição dos dados, seguida por testes de associação (análise bivariada) e análise multivariável utilizando regressão de Poisson com ajuste robusto da variância para amostragem complexa conforme modelo hierárquico de análise previamente estabelecido. Foi utilizada matriz de correlação para testar a colinearidade entre as variáveis. $\mathrm{Na}$ regressão foram consideradas como potenciais fatores de confusão as variáveis que na análise bivariada com o desfecho apresentaram valor $\mathrm{p}$ inferior a 0,20 .

Em $10 \%$ da população foi realizada uma entrevista para controle de qualidade com aplicação de pergunta-chave do questionário. Foi encontrada uma concordância de $95,8 \%$ e um índice Kappa de 0,92 (p < 0,001) para presença de cães/gatos no domicílio.

O projeto foi submetido e aprovado pelo Comitê de Ética em Pesquisa da Faculdade de Medicina da Universidade Federal de Pelotas. Para participar do estudo os sujeitos assinaram o termo de consentimento livre e esclarecido, foi garantido o direito a não participação e o sigilo em relação à identidade dos participantes.

\section{Resultados}

Foram investigados 1.558 domicílios, totalizando 9,6\% de perdas/recusas. Entre os domicílios, 59\% possuíam animais de estimação (cão e/ou gato), o número médio de moradores por domicílio foi de 2,8 e o de animais (cão e/ou gato) foi de 1,4. Considerando apenas os domicílios que possuem animais, a média de animais por domicílio foi de 2,4.

Do total de 2.185 animais, sendo 1.605 cães e 580 gatos, menos de $20 \%$ foi esterilizado, $72 \%$ dos animais receberam vermífugo nos últimos seis meses e somente $39 \%$ dos animais receberam vacinação antirrábica nos últimos 12 meses. Além disso, pouco mais de um terço $(36 \%)$ recebeu atendimento médico veterinário neste mesmo período. $\mathrm{O}$ acesso dos animais às áreas públicas, desacompanhados ou com o dono, mas sem o uso da guia, é comum a 26,7 dos animais domiciliados (Tabela 1).

Nos 918 domicílios com animais o controle de ectoparasitas foi realizado em $78 \%$. A aplicação de produtos diretamente nos animais foi usada em $49 \%$ dos domicílios, enquanto apenas $27 \%$ utilizaram algum tipo de controle também no ambiente. Nos domicílios onde os proprietários costumam passear com os animais, apenas um quarto relatou recolher as fezes do animal durante o passeio (Tabela 2).

A distribuição do escore de guarda responsável demonstra que $64 \%$ da população encontrou-se entre os pontos 5 e 9 do escore. Considerando que os sete critérios abordados no inquérito referem-se a cuidados essenciais, a pontuação inferior a 7 pontos significa ausência total de pelo menos um aspecto examinado, a pontuação de 7 a 13 pontos encontrada em $54 \%$ da população representa alguma preocupação com os itens investigados, ainda que de forma insatisfatória (Figura 1).

$\mathrm{Na}$ análise bruta, com exceção da variável presença de idoso no domicílio, todos os preditores avaliados apresentaram associação com o desfecho. Após a análise ajustada observou-se um maior valor de escore de posse em famílias de maior nível educacional e que moram em apartamento, com valores de $\mathrm{p}<0,001$ e $\mathrm{p}=$ 0,01 respectivamente. A renda familiar apresentou colinearidade com a escolaridade do chefe da família, sendo excluída da análise multivariável (Tabela 3). 
Quadro 1. Questionário utilizado para gerar o escore de posse responsável.

\begin{tabular}{|c|c|}
\hline Questões & Pontuação do escore \\
\hline Tem cachorro ou gato aqui na sua casa? & $\begin{array}{l}\text { Não }=0 \\
\text { Sim }=1\end{array}$ \\
\hline $\begin{array}{l}\text { Quantos cachorros? } \\
\text { Quantos gatos? }\end{array}$ & $\begin{array}{l}\text { Não faz parte do escore } \\
\text { Não faz parte do escore }\end{array}$ \\
\hline \multicolumn{2}{|l|}{$\begin{array}{l}\text { Destes animais, quantos não podem mais ter filhotes porque foram } \\
\text { castrados? }\end{array}$} \\
\hline $\begin{array}{l}\text { Desde “últimos } 12 \text { meses” pra cá, quantos destes animais foram } \\
\text { vacinados contra a raiva? }\end{array}$ & $\begin{array}{l}\text { Nenhum }=0 \\
\text { Alguns }=1 \\
\text { Todos }=2\end{array}$ \\
\hline $\begin{array}{l}\text { Desde “últimos } 12 \text { meses” pra cá, quantos destes animais foram } \\
\text { levados para consultar com veterinário pelo menos uma vez? }\end{array}$ & $\begin{array}{l}\text { Nenhum }=0 \\
\text { Alguns }=1 \\
\text { Todos }=2\end{array}$ \\
\hline $\begin{array}{l}\text { Desde “últimos seis meses” pra cá, vocês usaram algum produto no } \\
\text { animal, ou aqui na casa, para combater pulgas ou carrapatos? } \\
\text { - não, nenhum } \\
\text { - sim, nos animais } \\
\text { - sim, no domicilio } \\
\text { - sim, nos animais e na casa } \\
\text { - não se aplica } \\
\text { - ignorado }\end{array}$ & $\begin{array}{l}\text { Nenhum }=0 \\
\text { Nos animais ou no ambiente }=1 \\
\text { Nos animais e no ambiente }=2\end{array}$ \\
\hline $\begin{array}{l}\text { Desde "últimos seis meses” pra cá, quantos destes animais receberam } \\
\text { algum remédio contra vermes? }\end{array}$ & $\begin{array}{l}\text { Nenhum }=0 \\
\text { Alguns }=1 \\
\text { Todos }=2\end{array}$ \\
\hline $\begin{array}{l}\text { Quantos destes animais costumam sair de casa sozinho ou são } \\
\text { levados para passear sem coleira? }\end{array}$ & $\begin{array}{l}\text { Nenhum }=0 \\
\text { Alguns }=1 \\
\text { Todos }=2\end{array}$ \\
\hline $\begin{array}{l}\text { Esta pergunta deve ser feita somente em caso de domicílio com cão. } \\
\text { Nessas ocasiões em que o(s) animal(is) sai(em) pra passear e faz(em) } \\
\text { cocô na rua, a pessoa que está com ele... Ler opções. } \\
\text { - não recolhe o cocô } \\
\text { - às vezes recolhe } \\
\text { - sempre recolhe } \\
\text { - o animal não sai para passear } \\
\text { - não se aplica } \\
\text { - ignorado }\end{array}$ & $\begin{array}{l}\text { Não recolhe }=0 \\
\text { Às vezes }=1 \\
\text { Sempre }=2\end{array}$ \\
\hline
\end{tabular}

\section{Discussão}

De forma inovadora, foi realizado um estudo de base populacional na zona urbana de Pelotas para avaliar a qualidade dos cuidados com animais de estimação praticados pela população. O estudo revelou que a guarda responsável aumenta com a escolaridade do chefe da família e é maior en- tre os moradores de apartamento. A maioria dos domicílios investigados atendeu apenas à metade dos critérios propostos.

Não se identificou um instrumento padronizado para investigar o desfecho de "guarda responsável”. Assim, desenvolveu-se um questionário para este estudo que se propôs a identificar apenas aspectos da guarda relativos à saúde pú- 
Tabela 1. Prevalência dos indicadores de guarda responsável de cães e gatos $(\mathrm{N}=2.185)$ domiciliados na área urbana do município de Pelotas em 2012.

\begin{tabular}{lcc}
\hline \multicolumn{1}{c}{ Variável } & N & \% \\
\hline Esterilização & 418 & 19,1 \\
Vacinação antirrábica anual & 851 & 38,5 \\
Consulta médica veterinária nos & 796 & 36,4 \\
últimos 12 meses & & \\
$\begin{array}{l}\text { Controle de endoparasitas nos } \\
\text { últimos 6 meses }\end{array}$ & 1.579 & 72,3 \\
Livre acesso às vias públicas & 583 & 26,7 \\
\hline
\end{tabular}

Tabela 2. Indicadores de guarda responsável nos domicílios que têm animais $(\mathrm{N}=918)$ na área urbana do município de Pelotas em 2012.

\begin{tabular}{lrc}
\hline \multicolumn{1}{c}{ Variável } & N & \% \\
\hline Controle de ectoparasitas & 195 & 21,2 \\
$\quad$ Nenhum & 450 & 49,0 \\
Somente nos animais & 20 & 2,2 \\
$\quad$ Somente no ambiente & 252 & 27,5 \\
$\quad$ Nos animais e no ambiente & & \\
Recolhimento das fezes dos animais & & \\
em vias públicas* & 234 & 25,7 \\
$\quad$ Não recolhe & 34 & 3,7 \\
Ás vezes & 111 & 12,2 \\
Sempre & 522 & 56,9 \\
Animal não sai de casa & \\
\hline
\end{tabular}

blica e não a avaliações de saúde individual dos animais. $\mathrm{O}$ tamanho amostral superior ao estimado para a realização da pesquisa, o alto valor do Kappa e o baixo índice de perdas e recusas reforçam a validade dos achados. Além disso, o inquérito revelou dados inéditos para a região pesquisada, sendo um dos poucos estudos sobre o assunto utilizando amostra de base populacional.

Segundo a World Society for the Protection of Animals (WSPA), guarda responsável implica em cuidar da saúde física, psicológica e ambiental do animal, zelar pelo seu bem-estar, reduzir o potencial de agressões e prevenir riscos que este possa trazer à comunidade, tanto do ponto de vista individual quanto do coletivo ${ }^{8}$. Infecções por agressões de animais são comuns e, frequentemente, requerem tratamento extenso ou hospitalização ${ }^{13}$.

$\mathrm{O}$ estudo apontou que o atendimento médico veterinário e a vacinação contra a raiva nos últimos 12 meses entre animais domiciliados foi inferior a $40 \%$, ficando muito aquém da recomendação da OMS de $80 \%$ de cobertura vacinal mínima da população canina total ${ }^{14}$. A presença do vírus da raiva em diversos tecidos de morcegos reforça a possibilidade de transmissão dessa zoonose para animais domésticos, uma vez que quando infectados podem apresentar paralisia, ou mesmo, movimentos desconexos, tornando-

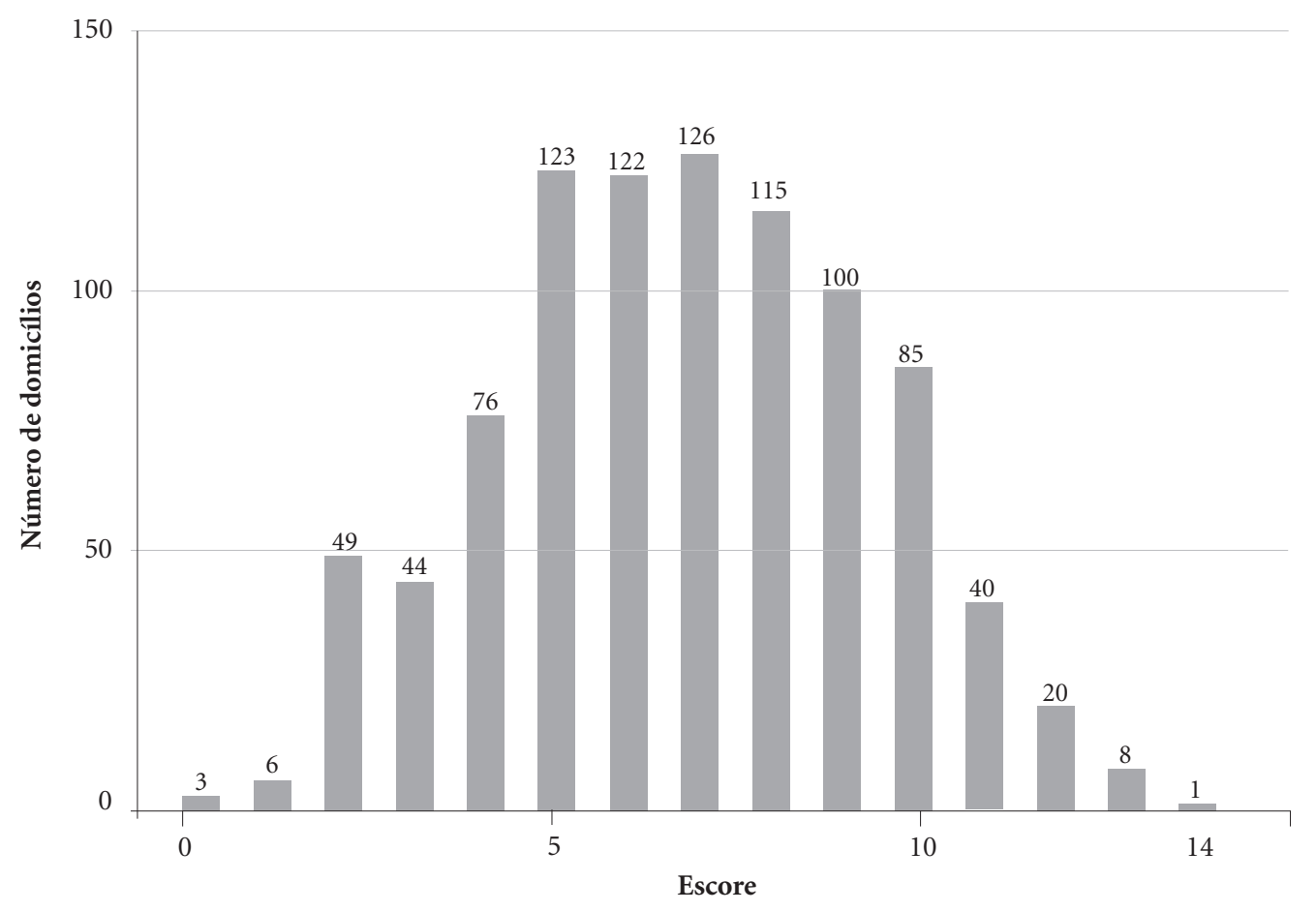

Figura 1. Distribuição do Escore de Guarda responsável na área urbana do município de Pelotas, 2012. 


\begin{tabular}{|c|c|c|c|c|c|c|}
\hline \multirow[t]{26}{*}{ 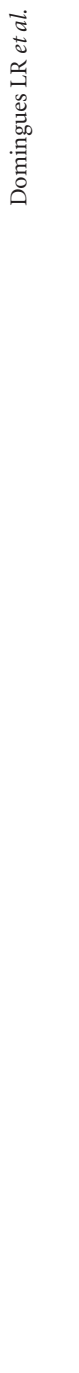 } & $\begin{array}{l}\text { Tabela 3. Análise de associação entre guarda responsá } \\
\text { Pelotas, } 2012 .\end{array}$ & le caracte & erísticas demǫ & gráficas da & população estu & dada. \\
\hline & \multirow[b]{2}{*}{ Variável } & \multirow[b]{2}{*}{$\begin{array}{l}\text { Escore } \\
\text { médio }\end{array}$} & \multicolumn{2}{|c|}{ Análise Bruta } & \multicolumn{2}{|c|}{ Análise Ajustada } \\
\hline & & & RP (IC95\%) & $\mathbf{p}$ & RP (IC95\%) & $\mathbf{p}$ \\
\hline & \multicolumn{6}{|l|}{ Primeiro nível } \\
\hline & Escolaridade do chefe da família & & & $<0,001$ & & $<0,001$ \\
\hline & Nenhuma ou até 3a série (ensino fundamental) & 5.7 & -- & & -- & \\
\hline & Ensino fundamental incompleto & 6.2 & $1,1(1,0-1,2)$ & & $1,1(1,0-1,2)$ & \\
\hline & $\begin{array}{l}\text { Ensino fundamental completo ou médio } \\
\text { incompleto }\end{array}$ & 6.4 & $1,1(1,0-1,2)$ & & $1,1(1,0-1,2)$ & \\
\hline & $\begin{array}{l}\text { Ensino médio completo ou nível superior } \\
\text { incompleto }\end{array}$ & 7.5 & $1,3(1,2-1,4)$ & & $1,3(1,2-1,4)$ & \\
\hline & Nível superior completo & 8.3 & $1,4(1,3-1,6)$ & & $1,4(1,3-1,6)$ & \\
\hline & Renda familiar (salários mínimos) & & & $<0,001$ & & \\
\hline & Até 1 & 5,7 & -- & & & \\
\hline & $1-3,99$ & 6,4 & $1,1(1,0-1,2)$ & & & \\
\hline & $4-6,99$ & 7,5 & $1,3(1,2-1,5)$ & & & \\
\hline & 7 ou mais & 7,8 & $1,4(1,2-1,5)$ & & & \\
\hline & \multicolumn{6}{|l|}{ Segundo nível } \\
\hline & Número de moradores & & & 0,03 & & \\
\hline & Até 3 & 6.9 & -- & & & \\
\hline & 4 ou mais & 6.6 & $1,0(0,9-1,0)$ & & & \\
\hline & Tipo de moradia & & & $<0,001$ & & 0,01 \\
\hline & Casa & 6.6 & -- & & -- & \\
\hline & Apartamento & 8.0 & $1,2(1,1-1,3)$ & & $1,1(1,0-1,2)$ & \\
\hline & \multicolumn{6}{|l|}{ Terceiro nível } \\
\hline & Presença de criança até 5 anos de idade & & & 0,03 & & \\
\hline & Não & 6.9 & -- & & & \\
\hline & Sim & 6.3 & $0,9(0,8-1,0)$ & & & \\
\hline
\end{tabular}

se presas fáceis para cães e gatos ${ }^{15}$, especialmente aqueles cujo acesso às ruas é irrestrito. Contudo, deve-se considerar a situação epidemiológica da região por não haver mais circulação viral da variante canina no Rio Grande do Sul, permanecendo o monitoramento sistemático.

A esterilização foi encontrada em apenas $19 \%$ dos animais, o que demonstrou a necessidade de incentivo aos proprietários sobre controle populacional destes animais. Segundo a OMS, as atividades isoladas de recolhimento e eliminação de cães e gatos não são efetivas para o controle da dinâmica destas populações, sendo necessário, portanto, atuar na causa do problema: a procriação animal sem controle ${ }^{8,16}$. Em 2010, uma pesquisa on-line sobre população animal, realizada na Austrália, encontrou um percentual de 80,4\% de esterilização ${ }^{17}$. É preciso considerar pelo menos dois fatores metodológicos ao comparar estes dados com nossos resultados: 1) a pesquisa foi desenvolvida em um país de renda bastante superior à do Brasil e que possui uma escolaridade média acima do nosso, sendo que especificamente esta pesquisa contou com participantes de escolaridade superior à da média australiana inclusive (30\% possuíam pós-graduação); 2) o fato de ser uma pesquisa que incluiu participantes de forma voluntária insere um grande viés de seleção na amostra e provavelmente os sujeitos entrevistados são diferenciados da população geral e se interessam mais por saúde animal.

O estudo mostrou que em Pelotas há um animal domiciliado para cada dois habitantes, superando a recomendação de um animal para cada oito habitantes, proposta pela Fundação Nacional de Saúde ${ }^{18}$, e a da OMS para países emergentes de um animal para cada sete habitantes ${ }^{14}$.

$\mathrm{Na}$ população estudada apenas $25 \%$ dos proprietários que levam seus animais a locais públicos recolhem as fezes dos mesmos. A contaminação de áreas públicas por fezes de animais constitui um problema de interesse coletivo, devido à possibi- 
lidade de transmissão de zoonoses, em especial, a toxocaríase (larva migrans visceral e ocular) e o "bicho geográfico" (larva migrans cutânea), doenças parasitárias causadas por larvas infectantes de Toxocara canis e Ancilostomídeos, respectivamente. Apesar da baixa letalidade entre humanos, essas doenças podem provocar diversas condições clínicas como dor, prurido e doenças como diarreia, anemias, entre outras, além de infecções secundárias por bactérias e fungos. Embora a infecção humana seja geralmente autolimitada, a irritação da pele e o prurido podem ocorrer por vários dias e ser de difícil diagnóstico ${ }^{13,19}$.

A guarda responsável de animais domésticos tem sido objeto de interesse da Organização Mundial da Saúde, sendo que em 2003 foi promovida no Rio de Janeiro a "Primeira Reunião Latino-Americana de Especialistas em Posse Responsável de Animais de Companhia e Controle de Populações Caninas"20. O atual reconhecimento dos benefícios que o convívio com animais de estimação traz à saúde humana demonstra que estudos devem considerá-lo como uma característica importante do domicílio ${ }^{4}$. A importância destes benefícios já vem sendo descrita em diferentes áreas da saúde ${ }^{21-24}$, entretanto, os animais também podem, em algumas situações, representar risco, porém o equilíbrio entre risco e benefício frequentemente não é feito de forma integrada. Assim, donos de animais ignoram orientações para se desfazer de um animal de estimação, mesmo quando apresentam doenças, como as alergias, que podem ser desencadeadas ou exacerbadas por animais. Além disso, idosos frequentemente rejeitam cuidados médicos por receio de serem admitidos em hospitais e forçados a separarem-se de seus animais ${ }^{25}$.

No ano de 2011, foi lançada a One Health Initiative que reforça o conceito de uma só saúde. É uma estratégia mundial para a expansão de colaborações interdisciplinares e comunicações entre todos os aspectos dos cuidados em saúde para os seres humanos, animais e o ambiente, representando um avanço em saúde para o século $\mathrm{XXI}^{26}$.

Os resultados deste estudo apontam para a necessidade de políticas públicas de educação que orientem a população sobre a guarda responsável de animais e da importância do controle populacional de ambas as espécies. A capacidade reprodutiva tanto de cães quanto de gatos torna necessária a criação de programas de controle populacional com a esterilização em massa acompanhada de políticas educacionais e de serviços gratuitos para a população de baixa renda.

\section{Colaboradores}

LR Domingues, JA Cesar, AG Fassa e MR Domingues participaram igualmente de todas as etapas de elaboração do artigo. 


\section{Referências}

1. Associação Nacional de Fabricantes de Alimentos para Animais de Estimação (Anfalpet). Mercado Pet Brasil. São Paulo: Anfalpet; 2011. [acessado 2011 jun 1]. Disponível em: http://abinpet.org.br/

2. Phillips-Donaldson D. Rising petfood powers. In: Phillips-Donaldson D, editor. Petfood Industry. Illinois: Watt Publishing; 2009. p. 22-29.

3. Wilson CC. The pet as an anxiolytic intervention. $J$ Nerv Ment Dis 1991; 179(8):482-489.

4. Hodgson K, Darling M. Zooeyia: An essential component of "One Health". Can Vet J 2011; 52(2):189-191.

5. Capuano DM, Rocha GM. Ocorrência de parasitas com potencial zoonótico em fezes de cães coletadas em áreas públicas do município de Ribeirão Preto, SP, Brasil. Rev. Brasileira de Epidemiologia 2006; 9(1):81-86.

6. Lima AMA, Alves LC, Faustino MAG, Lira NMS. Percepção sobre o conhecimento e profilaxia das zoonoses e posse responsável em pais de alunos do pré-escolar de escolas situadas na comunidade localizada no bairro de Dois Irmãos na cidade do Recife (PE). Cien Saude Colet 2010; 15(1):1457-1464.

7. Taylor LH, Latham SM, Woolhouse MEJ. Risk factors for human disease emergence. Philos Trans R Soc Lond B Biol Sci 2001; 356(1411):983-989.

8. World Health Organization (WHO). The control of neglected zoonotic diseases. Geneva: WHO, WSPA; 2005.

9. Westgarth C, Heron J, Ness AR, Bundred P, Gaskell RM, Coyne KP, German AJ, MacCune S, Dawson S. Family Pet Ownership during Childhood: Findings from a UK Birth Cohort and Implications for Public Health Research International. J Environ Res Public Health 2010; 7(10):3704-3729.

10. Downes MJ, Clegg TA, Collins DM, McGrath G, More SJ. The spatial distribution of pet dogs and pet cats on the island of Ireland. BMC Vet Res 2011; 7:28.

11. Barros AJD, Santos IS, Assunção MCF, Gigante D, Fassa AG, Marques M, Araújo C, Hallal PC, Facchini LA. O Mestrado do Programa de Pós-graduação em Epidemiologia da UFPel baseado em consórcio de pesquisa: uma experiência inovadora. Rev Brasileira de Epidemiologia 2008; 11(Supl. 1):133-144.

12. Instituto Brasileiro de Geografia e Estatística (IBGE). Censo Brasileiro 2010. Rio de Janeiro: IBGE; 2011.

13. National Association of State Public Health Veterinarians. Compendium of Measures to Prevent Disease Associated with Animals in Public Settings. MMWR Recommendations and reports 2009; 58(RR05):1-15. [acessado 2011 mar 1]. Disponível em: http://www.cdc. gov/mmwr/preview/mmwrhtml/rr5805al.htm

14. World Health Organization (WHO). Guidelines for dog population management. Geneva: WHO, WSPA; 1992.

15. Genaro G. Gato doméstico: futuro desafio para controle da raiva em áreas urbanas? Pesquisa Veterinária Brasileira 2010; 30(2):186-189.
16. São Paulo. Secretaria Estadual da Saúde. Programa de controle de populações de cães e gatos do Estado de São Paulo. Governo do Estado de São Paulo. 2009. [acessado 2011 mar 1]. Disponível em: http://www.saude. sp.gov.br/resources/ccd/publicacoes/publicacoes-ccd/ manuais-normas-e-documentos-tecnicos/manuaisnormasedocumentostecnicos1_-_manual_de_controle _de_populacoes_de_caes_e_gatos_no_estado_de sao_paulo_-_2009.pdf

17. Rohlf VI, Bennett PC, Toukhsati S, Coleman G. Why do even committed dog owners fail to comply with some responsible ownership practices? Anthrozoos 2010; 23(2):143.

18. Brasil. Ministério da Saúde (MS). Fundação Nacional de Saúde. Encontro Nacional dos Coordenadores de Zoonoses. Relatório Anual. Brasília: MS; 2002.

19. Vasconcellos MC, Barros JSL, Oliveira CS. Parasitas gastrointestinais em cães institucionalizados no Rio de Janeiro, RJ. Rev Saude Publica 2006; 40(2):321-323.

20. Souza MFA. Primeira Reunião Latino-americana de especialistas em posse responsável de animais de companhia e controle de populações caninas. In: Primeira Reunião Latinoamericana de especialistas em posse responsável de animais de companhia e controle de populações caninas; de 01 a 03 de setembro de 2003. Rio de Janeiro; 2003.

21. Kramer SC, Friedmann E, Bernstein PL. Comparison of the Effect of Human Interaction, Animal-Assisted Therapy, and AIBO Assisted Therapy on Long-Term Care Residents with Dementia. Anthrozoos 2009; 22(1):43-57.

22. Laun L. Benefits of pet therapy in dementia. Home Healthc Nurse 2003; 21(1):49-52.

23. Walsh F. Human-Animal Bonds I: The Relational Significance of Companion Animals. Family Process 2009; 48(4):462-480.

24. Ham SA, Epping J. Dog walking and physical activity in the United States. Prev Chronic Dis 2006; 3(2):47.

25. McNicholas J, Gilbey A, Rennie A, Ahmedzai S, Dono JA, Ormerod E. Pet ownership and human health: a brief review of evidence and issues. BMJ 2005; 331(7527):1252-1255.

26. One Health Initiative. One Health Initiative will unite human and veterinary medicine. [acessado $2012 \mathrm{mar}$ 1]. Disponível em: http://www.onehealthinitiative. com/

Artigo apresentado em 14/08/2013

Aprovado em 10/10/2014

Versão final apresentada em 14/10/2013 